Benjamin Böhm, Christof Heeger, Isaac Boxx, Wolfgang Meier, Andreas Dreizler, Time-Resolved Conditional Flow Field Statistics in Extinguishing Turbulent Opposed Jet Flames using Simultaneous Highspeed PIV/OH-PLIF, Proc. Combust. Inst. 32 (2), (2009) 1647-1654

The original publication is available at www.elsevier.com

http://dx.doi.org/10.1016/j.proci.2008.06.136 


\title{
Time-Resolved Conditional Flow Field Statistics in Extinguishing Turbulent Opposed Jet Flames using Simultaneous Highspeed PIV/OH- PLIF
}

\author{
B. Böhm ${ }^{1}$, C. Heeger ${ }^{1}$, I. Boxx², W. Meier ${ }^{2}$, A. Dreizler ${ }^{1}$ \\ 1: Fachgebiet Energie- und Kraftwerkstechnik, Technische Universität Darmstadt \\ Petersenstrasse 30, D - 64287 Darmstadt, Germany \\ 2: Institut für Verbrennungstechnik, Deutsches Zentrum für Luft- und Raumfahrt (DLR), \\ Pfaffenwaldring 38-40, D-70569 Stuttgart, Germany
}

Corresponding author: Andreas Dreizler, Petersenstrasse 30, D - 64287 Darmstadt, Germany, Fax:+49 6151 166555, e-mail: dreizler@ekt.tu-darmstadt.de

\section{Colloquium \\ Turbulent Combustion}

\section{Paper length}

o Main text (Introduction $\rightarrow$ Acknowledgements): 3636

o Equations: 0

o Nomenclature: 0

o References: $(19+2) \times 2.3 \times 7.6=368$

o Tables: $(0+2) \times 7.6 \times 4=0$

o Figures \& figure captions:

o Fig. 1: $(43+10) \times 2 \cdot 2+7=124$

o Fig. $2:(73+10) \times 2 \cdot 2+33=216$

o Fig. 3: $(177+10) \times 2 \cdot 2 \times 2+41=864$

o Fig. 4: $(99+10) \times 2.2 \times 2+37=517$

o Fig. 5: $(71+10) \times 2.2+21=193$

o Fig. 6: $(86+10) \times 2 \cdot 2+18=229$

o Total figures:2149

\section{Total count: 6147}

Herewith we affirm to pay color reproduction charges for 3 figures (this includes figure 3,4 and figure 5). 


\begin{abstract}
Time resolved particle image velocimetry (PIV) and planar laser induced fluorescence (PLIF), both at $5 \mathrm{kHz}$, were applied simultaneously on extinguishing turbulent opposed jet flames. This repetition rate allowed tracking of transient extinction events in turbulent combustion. The additional information acquired about time history enabled a study of the evolution of vortex-flame interactions leading to extinction from individual events. A newly introduced multidimensional conditioning technique to avoid spatial- and temporal-smearing of important flow field information was developed in order to compare individual extinction events in a meaningful, statistical manner. The conditional statistics show that vortices tend to align around the flame and generate regions of high strain in the region where the flame is about to extinguish.
\end{abstract}

Keywords

High speed laser diagnostics, combined PIV/OH-PLIF imaging, time resolved conditional flow field statistics, turbulent opposed jet flames, extinction 


\section{Introduction}

The mutual interaction of turbulent flows and chemical reactions is a major challenge in combustion science. Generic configurations without additional complications, such as flame holders or wall boundary layers, provide well accepted platforms to study these processes and validate models implemented in numerical simulations. Examples of generic configurations burning in a non-premixed mode are piloted and swirled jet flames (summarized by Barlow [1]) and turbulent opposed jet flames [2]. A variety of turbulent non-premixed flames have been extensively characterised using multiple laser-based diagnostics. Much of this data, including information on the boundary conditions, flow and scalar fields, is accessible on the TNF webpage [3,1].

The ultimate consequence of the impact of very intense turbulence on chemical reactions is flame extinction. Valuable insight into transient thermo-kinetic states such as extinction, which deviate significantly from chemical equilibrium have been provided through multiscalar diagnostics based on one-dimensional Raman/Rayleigh spectroscopy [4], polarized/depolarized Rayleigh and CO laser-induced fluorescence (LIF) imaging [5]. Results of these measurements were reported mainly in the form of either scatter plots or mean and variances of scalars, including scalar dissipation rate (SDR). As repetition rates of these diagnostics were at low frequencies the time lag between consecutive samples exceeded integral time scales of the turbulent flames by orders of magnitude. Hence sampling was statistically independent. Furthermore, statistical databases were limited to, at best, a few thousand realisations per probe volume. Consequently, statistical errors at the periphery of probability density functions (PDF) may be significant. In the case of SDR measurements, where the highest measured values are related primarily to flame extinction events, the statistical errors are an issue. Another challenge in SDR-measurements is the trade-off between experimental 
noise and spatial resolution [6,7]. Noise causes a positive bias error leading to an "apparent SDR” [8]. Reducing this bias, however, by increasing probe volume size is prohibitive as the smallest length scales may be no longer spatially resolved [9]. One way to circumvent these experimental issues in turbulent flame measurements is to generate "controlled unsteadiness" to allow for phase-locked measurements. The opposed jet configuration, where individual vortices have been generated travelling through the reaction zone, is a prominent example of such investigations $[10,11,12]$. Given the simplicity of the flow field even direct numerical simulations (DNS) of these flames are possible [13].

An interesting finding of Katta et al. (13, and references therein) was that unsteady flames can resist strain up to twice the extinction limit of laminar flames. This was attributed to the time lag between diffusive and convective processes. Even SDR was believed to be an insufficient measure of extinction when chemical kinetics, rather than diffusive processes, were ratelimiting (“chemistry-limited situation”). These authors proposed a new variable, defined as the ratio of the strain rate to the rate of change in flame temperature to characterize the unsteady quenching process (see equation 3 in Katta et al. [13]).

Despite offering valuable insight into differences between the extinction processes occurring during steady and unsteady flow conditions, single (or dual) vortices are very different from real turbulence. Studying turbulent opposed jet flames (TOJ) is an important step towards understanding fully turbulent flows, which contain a wider spectrum of spatial and temporal length scales. From a practical standpoint, there are also definite benefits to this configuration: starting from stable conditions, flames can be operated gradually closer to extinction by increasing the momentum of the flows issuing from the opposed nozzles. Using identical momentum of the impinging flows, global extinction begins in the vicinity of the 
mean stagnation point. This is evident from temporally resolved chemiluminescence imaging. Consequently, diagnostics can be focused on a restricted volume close to the mean stagnation point. The configuration is well suited for comparisons to highly resolved numerical simulations because the computational domain can be restricted to a much smaller volume than jet flames, for example. The main disadvantage of turbulent opposed flames is the relatively low Reynolds-numbers at which extinction occurs and that the turbulence is not fully developed (“young turbulence” [14]).

To complement existing knowledge about extinction of turbulent opposed jet flames [2] in a previous study the turbulent flow field was measured from the viewpoint of flame-fixed coordinates (conditional instantaneous velocities). This was accomplished using simultaneous particle image velocimetry (PIV) and OH-PLIF measurements reported in Böhm et al. [15]. The scope of this paper is to include time as an important dynamic parameter of extinction. This was accomplished by increasing the acquisition rate of the simultaneous PIV/OH-PLIF measurements to $5 \mathrm{kHz}$ and thus acquiring statistically dependent, combined velocity and scalar field data over a significant temporal history. This allowed temporal tracking of velocity fields adjacent to the unsteady reaction zone prior to extinction. A new procedure was developed to conditionally average flow field properties such as strain rates and vorticity prior to extinction. Findings support those of previous studies which conclude strain rates alone are insufficient to parameterize extinction for unsteady flow conditions.

\section{Experimental set up}

\subsection{Planar laser-induced fluorescence of $\mathrm{OH}$}

The hardware setup for this experiment is shown in Fig. 1. The OH-PLIF system (more thorough described in Kittler et al. [16], used a modified conventional frequency-doubled dye 
laser (Radiant Dyes) was used to excite the $Q_{1}(9)$ line of $O H$ within the $A^{2} \Sigma-X^{2} \Pi(1-0)$ band. The dye laser was pumped by a frequency-doubled Nd:YLF laser based on an edge-pumped slab design (Edge Wave IS8II-E), at $523 \mathrm{~nm}$. The repetition rate of the pump laser was set to $5 \mathrm{kHz}$ (3.4 mJ/pulse). At $5 \mathrm{kHz}$ the daily-routine single-pulse energies at $283 \mathrm{~nm}$ were $\sim 50 \mu \mathrm{J}$ (4 ns pulse duration) with pulse-to-pulse fluctuation of $\sim 18 \%$ rms. The UV-beam profile was smooth and was formed into a sheet $16 \mathrm{~mm}$ high and $200 \mu \mathrm{m}$ thick.

Fluorescence was monitored with a CMOS camera (HSS5, LaVision) equipped with an achromatic UV-lens (Halle, f=100 mm, f\# 2), a custom-fabricated bandpass filter (300320nm) and a two-stage, lens-coupled image intensifier (LaVision, HS-IRO). The architecture of the CMOS camera's 2.6GB of on-board memory uses a self-overwriting cyclic loop for continuous image acquisition. One therefore has the option of post-triggering an acquisition sequence from after a flow field event and storing images acquired prior it. For unpredictable events such as flame extinction, this feature offers a significant advantage over pre-triggered data acquisition systems [17]. Utilizing manual, post-extinction triggering allowed us to capture extinction events in the measurement runs with a $100 \%$ success rate. The partialframe image size of the camera at $5 \mathrm{kHz}$ was $768 \times 768$ pixels, resulting in 4000 frames per recording (0.8 s acquisition time).

\subsection{Particle image velocimetry}

The hardware setup for this experiment is shown in Fig. 1. Two components of the instantaneous velocity field were measured simultaneously with the relative $\mathrm{OH}$ distributions. For this purpose a frequency-doubled, dual-cavity $\mathrm{Nd}: \mathrm{YVO}_{4}$ slab laser (IS4II-DE, Edge Wave) operated at $532 \mathrm{~nm}$ was used. The repetition rate was set to $5 \mathrm{kHz}$ with a pulse to pulse separation of $100 \mu \mathrm{s}$. Mie scattering was imaged with a second CMOS camera (HSS5, 
LaVision). The detection angle was $80^{\circ}$ in forward scattering, to allow for $90^{\circ} \mathrm{OH}-\mathrm{PLIF}$ detection at the same side of the flame. The images of both cameras were superimposed in a post-processing step based on a backlit spatial calibration target. Two independent seeders provided $1 \mu \mathrm{m}-\mathrm{MgO}$-particles for the air and fuel flows. The laser sheets of the PLIF and PIV systems were overlapped spatially using dichroic mirrors. The $\mathrm{OH}$ excitation pulse was delivered 50 $\mu$ s after the first PIV pulse (i.e. halfway between PIV pulses) of each cycle.

Data processing was based on a PIV-algorithm presented in Böhm et al. [15]. Prior to the calculation of the differential properties, a proper orthogonal decomposition (POD) of the considered data set was conducted, as it is a very effective scale decomposition for visualising vortices [18]. This was band-pass filtered before the velocity map was reconstructed through a reverse transformation. The spectrum was cut off beyond mode 20 because the higher modes carried mainly noise. This resulted in a smoother velocity field. Strain was calculated from this velocity field, while for vorticity the first mode, which carries the information of the mean flow field, was also cut off.

\subsection{Image post-processing}

Specific spatiotemporal points where global extinctions begin are unpredictable. Averaging flow and scalar field properties using the laboratory-fixed co-ordinate system therefore smears important details. To overcome this limitation a new procedure utilizing multidimensional conditional co-ordinates is proposed.

Figure 2 summarizes the procedure and shows how averaging of different individual extinction events was accomplished. For each individual extinction event, the frame where the flame is first breached was selected as time zero (i.e. the start of global extinction). This frame 
was defined as an "extinction-fixed" time or $\mathrm{t}^{*}=0 \mathrm{~ms}$. In the second step, the origin of the radial co-ordinate was shifted to the centre of the initial flame breach (observed at $t^{*}=0 \mathrm{~ms}$ ). In the third and final step, instantaneous $\mathrm{OH}$ distributions prior to the start of extinction were used to determine the intermittent flame location for each individual frame. The location where the lower $\mathrm{OH}$ contours cut the vertical axis at $\mathrm{r}^{*}=0 \mathrm{~mm}$ was defined as the origin of a flame-fixed axial co-ordinate $\mathrm{z}^{*}$. This procedure resulted in spatiotemporal co-ordinates conditioned on both the nonstationary flame $\left(\mathrm{z}^{*}\right)$, start of extinction in radial direction $\left(\mathrm{r}^{*}\right)$, and time $\left(\mathrm{t}^{*}\right)$. This multidimensional conditioning in space and time allowed a rigorous statistical analysis of flow quantities prior to and at the point of extinction. Even though extinction begins randomly in the vicinity of the stagnation point and randomly in time, by multidimensional conditioning the data were transformed such that the onset of extinction was centered in the new coordinate system for each individual event.

\subsection{Turbulent opposed jet flame: a generic benchmark}

The experiments were performed in a turbulent partially-premixed methane/air flame close to extinction. The burner, sketched in Fig. 1, is described in greater detail in Geyer et al. [2]. In brief, a $30 \mathrm{~mm}$ diameter air stream is opposed by an equivalent diameter methane/ air mixture $(\phi=2.0)$. Both streams had equal momentum. A flammable mixture was therefore formed at the half-nozzle distance $\mathrm{H} / 2=15 \mathrm{~mm}$. The turbulence intensities of both streams were increased using perforated plates (blockage $45 \%$, $\mathrm{d}=4 \mathrm{~mm}$ holes) upstream of the jet-nozzles. The exit Reynolds number of the air stream was 6650. Under these conditions the nearextinction flame was seen to persist for (on average) 30sec. The opposed jets were surrounded by a Nitrogen co-flow to prevent mixing with ambient air and also to shield the flame from disturbances from the laboratory environment. 
A typical acquisition sequence proceeded as follows. The flame was ignited using a torch and a cyclic data acquisition loop was initiated. The burner was operated for at least $5 \mathrm{~s}$ to ensure steady-state conditions prior to extinction and then observed by an operator. When global extinction was observed, the operator manually released a TTL trigger to the camera to stop new data acquisition and begin storing the previously acquired frames. By this technique we typically acquired at least 1000 measurements before and after the extinction event, allowing a full temporal reconstruction of it in post-processing.

\section{Results}

Using the technique described above, 14 individual extinction events were recorded. Each experiment run acquired $0.8 \mathrm{~s}$ of measurement at a constant, $5 \mathrm{kHz}$ repetition rate, however the analysis here focuses only on the few ms prior to the first breach in the flame sheet.

Figure 3 shows two individual measurement sequences prior to extinction, spanning from $\mathrm{t}^{*}=-4.4$ to $+0.8 \mathrm{~ms}$ (N.B. frame-to-frame intervals in this figure are not identical). Instantaneous $\mathrm{OH}$ contours are represented by black lines. Imposed on the instantaneous velocity vectors are the POD-filtered out-of-plane vorticity and the absolute values of the strain, calculated from the two velocity components. Up to approximately $4 \mathrm{~ms}$ prior to extinction regular patterns were not observed in the vorticity or strain fields. Turbulence appeared as stochastically distributed eddies in space and time. Starting from approximately $t^{*}=-4 \mathrm{~ms}$, characteristic flow field structures observed to appear and peak strain and vorticity values increased progressively.

Inspection of 14 individual data sequences revealed two classes of extinction events. For 8 cases we observe two counter-rotating vortices from the fuel side convecting towards the lower $\mathrm{OH}$ contour (denoted as Class I, shown in Fig. 3a). These eddies align below the flame. 
By expansion due to heat release at the flame front the vortices distorted radially. They lingered in approximately the same spatial location as their vorticity rapidly increased. The centers of rotation of each eddy stabilized approximately 2-3 $\mathrm{mm}$ below the lower $\mathrm{OH}$ contour and were radially separated from each other by 5-6 mm. The magnitude of the radial separation was likely driven by the lengthscale of the turbulence enhancement plates (interhole spacing: $5 \mathrm{~mm}$ ). The counter-rotating vortices transported fuel that impinged perpendicularly (in a jet-like manner) upon the flame front to produce greater compressive and extensive strains. Strain increased with vorticity until $t^{*}=-0.8 \mathrm{~ms}$. By that time the $\mathrm{OH}-$ contour began to contract and was finally breached at $t^{*}=-0$ ms. Convection pushed the flame tips in opposing radial directions. After the breach occurs, convection appears to dominate the flame's radial propagation. In rare cases (not shown here), flame breaches were observed to occur in the vicinity of the mean stagnation point and then collapse and convect outwards to allow the reestablishment of a self-sustaining turbulent flame.

The remaining 6 extinction events were classified as Class II events. In general they showed less regularity than Class I extinctions. Figure 3b shows one of six events. A counter-rotating vortex pair originating from the air side and a single vortex from the fuel side surround the flame starting from $t^{*=-5}$ to -4 ms. These vortices were smaller and more distorted than in Fig. 3a. During the vortex-flame interaction the flame becomes significantly distorted. In a manner reminiscent of Class I events however, a region of high strain developed just below the lower OH contour. For both Class I and II type events, peak strain values are observed to be of the same order of magnitude. In Class II events flame tips resisted the radial direction convection for a longer period. This could be caused either by different local mixture fraction distributions or by lower instantaneous radial velocity at the location of the flame tip. No experimental evidence of the former is present, however evidence of the latter was observed at the left side of the flame between $\mathrm{t}^{*}=0$ and $0.8 \mathrm{~ms}$, as shown in Fig. $3 \mathrm{~b}$. 
These single temporal sequences show that vorticity does play a vital, but not exclusive, role in understanding extinction of turbulent opposed jet flames. A few ms prior to extinction, multiple vortices acting coherently generate strain over extended regions close by the flame. According to Lemaire et al. [10] peak strain was identified to be a significant controlling parameter. However, in Katta et al. [13], based on numerical simulations, unsteady flames were observed to resist strain twice the extinction level of laminar flames. Single sequences shown in Fig. 3a and b exhibit strain increased locally up to $1500 \mathrm{~s}^{-1}$. To draw further conclusions, extinction progress must be averaged appropriately. This is accomplished by multidimensional conditioning in space and time, as detailed in the previous section.

Figure 4 shows the conditional average of POD-filtered out-of-plane vorticity and twocomponent strain for all 14 experiment runs (I+II, left), as well as for Class I (middle) and Class II cases (right) separately. The black lines denote the mean $\mathrm{OH}$ contours. Vorticity fields are presented for $t^{*}=-4.4,-2.4$ and $-0.4 \mathrm{~ms}$ whereas strain is shown only for $\mathrm{t}^{*}=-0.4 \mathrm{~ms}$. Conditional averages of the temporal evolution of both vorticity and strain for Class I as well as for Class II support the conclusions drawn already from the single events. Class I showed primarily strong counter-rotating vortex pairs from the fuel side, causing a distinct region of peak strain values. Class II showed less regular vortices predominantly from the air side. The shape of individual vortices and regions of high strain differed for individual extinction sequences. Consequently, peak values in the conditional mean data were reduced by a factor of approximately two compared to individual progressions. Despite the differences in the conditionally averaged vorticity fields areas of maximum strain were observed to align in all cases (I+II) slightly below the lower $\mathrm{OH}$ contour. 
The measured strain components can only give an approximation of the principal strain rates because the flow measurements are limited to two components of the flow field. The absolute strain given in this work includes only two components of strain while the flame experiences an additionally extensive component out of the measurement plane. Because the flame is approximately horizontal with slight inclinations caused by eddies it can be assumed to be normal to the measurement plane and thereby the out of plane component is approximately a principal strain rate [19]. The conditional average of the axial strain component acting compressive (negative) and radial component extensive (positive) are shown in figure 5 for all 14 runs (I+II). Two local maxima of extensive strain begin to form at $\mathrm{t}=-4.4 \mathrm{~ms}$ in the region of the lower $\mathrm{OH}$-contour $\sim 1-2 \mathrm{~mm}$ radially to the right and left of the point were extinction is going to occur $\left(\mathrm{z}^{*}=0, \mathrm{r}^{*}=0 \mathrm{~mm}\right)$ while a maximum of compressive strain is formed inbetween these peaks at the lower $\mathrm{OH}$-contour just below the region where the $\mathrm{OH}$ layer is breached first.

For a more quantitative representation of the conditional averages, Fig. 6 shows temporal evolutions of strain and vorticity recorded at various monitor points. The spatial location of each monitor point is shown in Fig. 4. The temporal traces proceed only up to $t^{*}=-0.2 \mathrm{~ms}$ as the definition of $z^{*}$ is based on continuous $\mathrm{OH}$ contours. MP1 corresponds to the origin of the newly introduced conditional co-ordinate system. MP2 is at the maximum of the conditionally averaged vorticity and MP3 is at maximum strain (compare Fig. 4, left column). Independent of classification, at MP1 and MP3 the conditional average of the strain was constantly $400 \mathrm{~s}^{-1}$ up to $5 \mathrm{~ms}$ prior to extinction. These early times correspond to statistically stationary turbulence. Strain increased from a conditional average of $400 \mathrm{~s}^{-1}$ at $\mathrm{t}^{*}=-4.4 \mathrm{~ms}$ to $700 \mathrm{~s}^{-1}$ (MP1) and $800 \mathrm{~s}^{-1}$ (MP2) at $\mathrm{t}^{*}=-1.4 \mathrm{~ms}$. The conditional average subsequently decreased slightly until the flame breached. Minimum and maximum values of conditional strain are shown by bars in Fig 6d. These bars do not indicate error ranges but rather the variability due 
to randomness of the turbulent flow field. In contrast to strain at MP1, conditional vorticity remained close to zero at all times. However, at MP2 the absolute value of conditional vorticity increased in a similar manner as the strain until it reached a plateau at $t^{*}=-1.4 \mathrm{~ms}$ (I+II). Differences in vorticity between Classes I and II were observed. For Class II, absolute values still increased, whereas in Class I, vorticity decreased.

MP1 is the location where the flame is first breached. Only conditionally averaged strain increased at this location. This indicates that for this type of turbulent flame, while vorticity acts to generate strain at distinct locations close to the flame, the magnitude of the vorticity is not as important as that of the strain for determining extinction. Traces of conditionally averaged strain pass a weak maximum at $\mathrm{t}^{*}=-1.4 \mathrm{~ms}$. This is a clear indication that peak strain values alone are not an appropriate measure to determine extinction for these unsteady flame conditions.

This finding is in agreement with Katta et al. [13]: Strain describes the flow structure. If strain is generated faster than diffusion time-scales, diffusion is delayed with respect to velocity imposition. Consequently non-equilibrium flow may occur, as described in Katta et al. [13]. During non-equilibrium situations, either of the two following mechanisms might be responsible for final extinction:

1) An increased flux of reactants into the reaction zone cools the flame, reduces reaction rates and promotes extinction.

2) The local Damköhler number will be decreased, also promoting extinction.

Increasing strain creates large scalar gradients. These gradients should reveal themselves by high values of scalar dissipation rate (SDR). Unfortunately, the temporal evolution of SDR conditioned to regions of high strain was not measurable in this experiment. Therefore, the hypothesis from Katta et al. [13] that even SDR is an insufficient measure of extinction in 
unsteady flames and for chemical non-equilibrium conditions (chemistry- rather than diffusion-limited conditions) could not be evaluated experimentally.

\section{Conclusions}

Previous experimental studies using simultaneous PIV/OH-PLIF on a turbulent, opposed jet flame were extended from $1 \mathrm{~Hz}$ to $5 \mathrm{kHz}$. This enabled the acquisition of statistically dependent datasets and allowed for tracking of the evolution of vortex-flame interactions in real time. Previous studies of PDFs of scalar dissipation rate (SDR) have displayed statistical errors at the peripheries, which are exactly the regions of the PDF most closely associated with extinction (as high SDRs are believed to cause extinction). The technique employed in this study allows one to focus specifically on the small fraction of measurement time directly relevant to extinction and thus vastly increases the accuracy, resolution and physical insight of experiments relating to this important phenomenon.

For the flame studied, two classes of flow structure leading to global extinction were identified. The newly introduced technique of multidimensional conditioning used new criteria to compare individual extinction events in a meaningful, statistical manner and avoid smearing important spatiotemporal information. It was found that turbulent vortices generate strain close to the flame and that extinction occurred when the resulting increase in strain exceeded critical values for sufficient time. Although strain was a more important measure for extinction than vorticity, peak strain alone was not a sufficient measure to predict extinction.

\section{Acknowledgments}

Financial support by Deutsche Forschungsgemeinschaft (SFB568, project B1) and Graduiertenkolleg 1344/1 is gratefully acknowledged. 


\section{References}

R.S. Barlow, Proc. Combust. Inst. 31 (2007) 49-75.

D. Geyer, A. Kempf, A. Dreizler, J. Janicka, Combust. Flame 143 (2005) 524-548.

TNF Workshop web site: http://public.ca.sandia.gov/TNF/abstract.html

$4 \quad$ R.S. Barlow, C.D. Carter, R.W. Pitz, in: K. Kohse-Höinghaus, J.B. Jeffries (Eds.), Applied Combustion Diagnostics, Taylor and Francis, New York, 2002, p. 384.

5 J.H. Frank, S.A. Kaiser, M.B. Long, Combust. Flame 143 (2005) 507-523.

6 D. Geyer, 1D-Raman/Rayleigh experiments in a turbulent opposed-jet, Dissertation, TU Darmstadt, 2004.

7 G.-H. Wang, N.T. Clemens, R.S. Barlow, P.L. Varghese, Meas. Sci. Technol. 18 (2007) 1287-1303.

$8 \quad$ S.P. Nandula, T.M. Brown, R.W. Pitz, Combust. Flame 99 (1994) 775-783.

9 J.C. Wyngaard, J. Sci. Instrum. 2 (1969) 983-987.

10 A. Lemaire, T.R. Meyer, K. Zähringer, J.R. Gord, J.C. Rolon, Exp. Fluids 36 (2004) 3642.

11 V. S. Santoro, D.C. Kyritsis, A. Gomez, Proc. Combust. Inst. 28 (2000) 1023-1030.

12 T.R. Meyer, G.J. Fiechtner, S.P. Gogineni, J.C. Rolon, C.D. Carter, J.R. Gord, Exp. Fluids 36 (2004) 259-267.

13 V.R. Katta, T.R. Meyer, J.R. Gord, W.M. Roquemore, Combust. Flame 132 (2003) 639651.

14 H. Tennekes, J.L. Lumley, A first course in turbulence, MIT Press, Cambridge, Massachusetts, 1972.

15 B. Böhm, D. Geyer, A. Dreizler, K.K. Venkatesan, N.M. Laurendeau, M.W. Renfro, Proc. Combust. Inst. 31 (2007) 709-718.

16 C. Kittler, A. Dreizler, Appl. Phys. B 89 (2007) 163-166.

17 C.F. Kaminski, M.B. Long, in: K. Kohse-Höinghaus, J.B. Jeffries (Eds.), Applied Combustion Diagnostics, Taylor and Francis, New York, 2002, p. 224.

18 R.J. Adrian, K.T. Christensen, Z.-C. Liu, Exp. In Fluids 29 (2000) 275-290.

19 Rehm, J. E. and N. T. Clemens (1999), AIAA-99-0676. 


\section{Figure captions and figures}

Fig. 1 Sketch of the experimental setup

Fig. 2 Exposure of data post processing for multidimensional conditioning. Flame contour/ velocity maps are recorded over thousands of frames including the time history prior to the first breach leading to global extinction

Fig. 3 Two individual sequences of extinction. Vorticity and strain are superimposed by false colors in top of the in-plane velocity vectors. Look-up tables in all cases are identical. Instantaneous locations of the $\mathrm{OH}$ contours are presented by black lines.

Fig. 4 Figure 4 Conditional averages of vorticity and strain for all events (I+II), class I and class II events at distinct times prior to extinction

Fig. 5 Conditional averages of axial strain (acting compressive) and radial strain (extensive) for all events (I+II).

Fig. 6 Temporal traces of conditional averaged vorticity and strain at monitor points (MP) assigned in Fig. 4. 


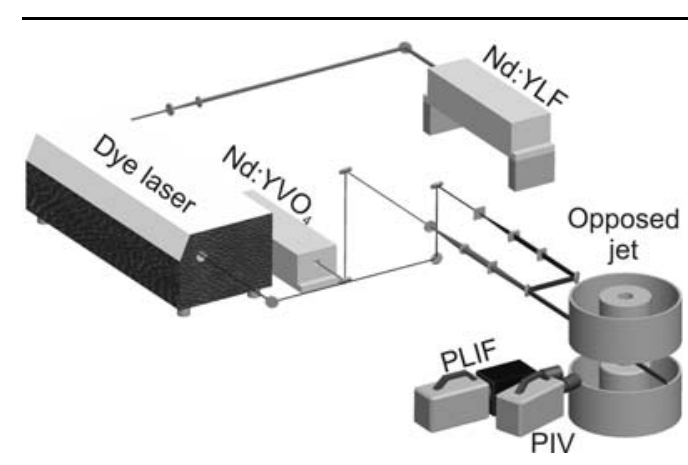

Fig. 1.

Sketch of the experimental setup.

Single-column width (b/w) 


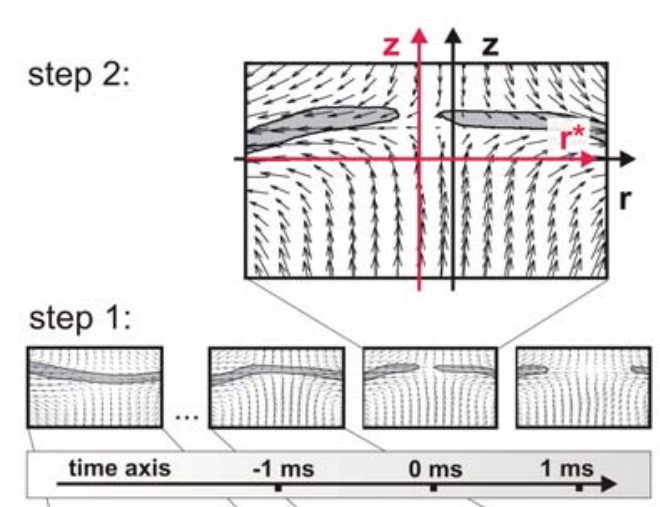

step 3:

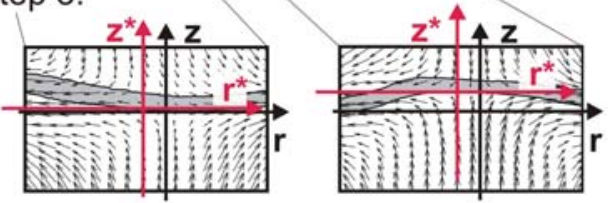

Fig. 2.

Exposure of data post processing for multidimensional conditioning. Flame contour/ velocity maps are recorded over thousands of frames including the time history prior to the first breach leading to global extinction.

Single-column width (b/w) 


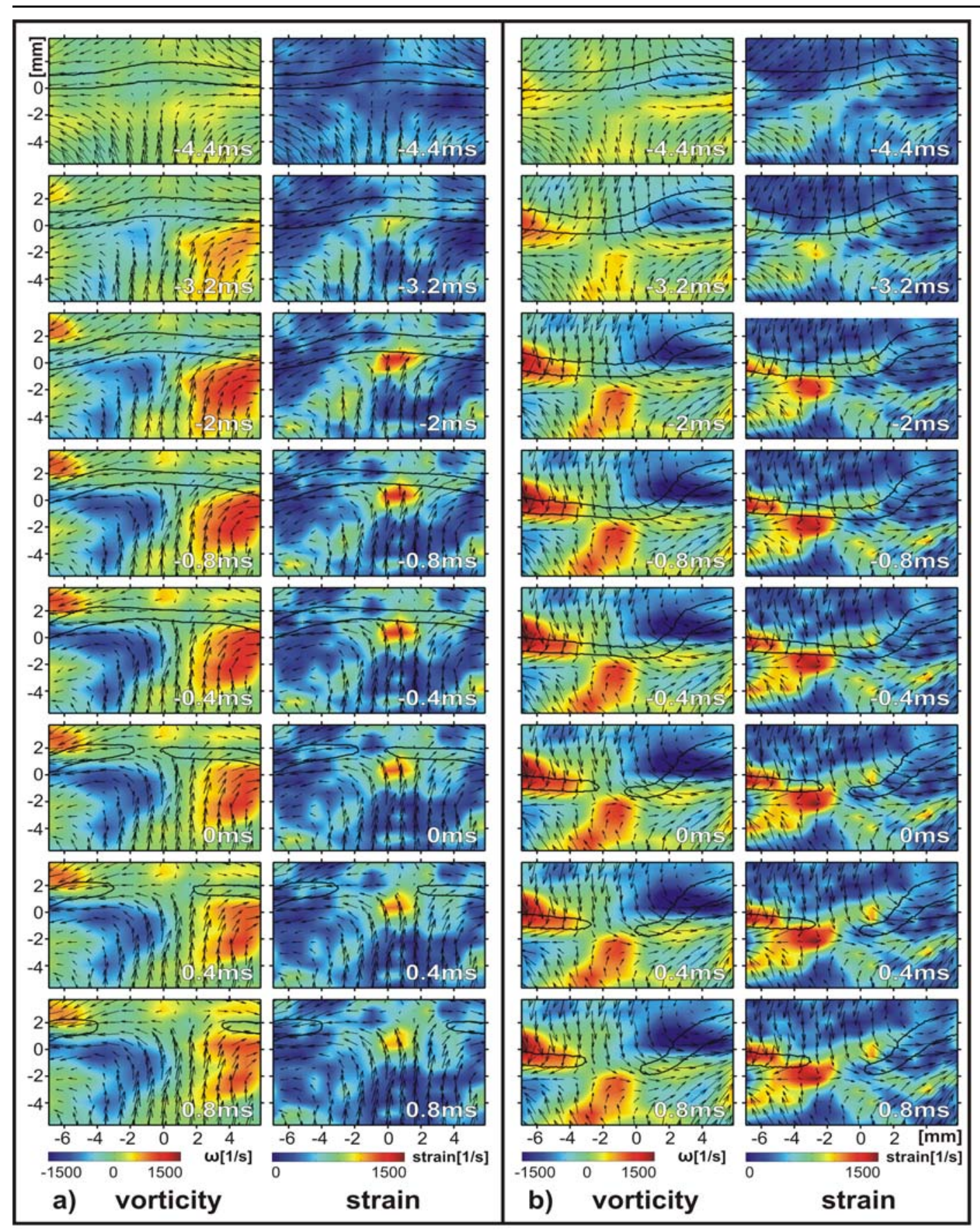

Fig. 3.

Two individual sequences of extinction. Vorticity and strain are superimposed by false colours in top of the in-plane velocity vectors. Look-up tables in all cases are identical. Instantaneous locations of the $\mathrm{OH}$ contours are presented by black lines.

Double-column width (color print) 

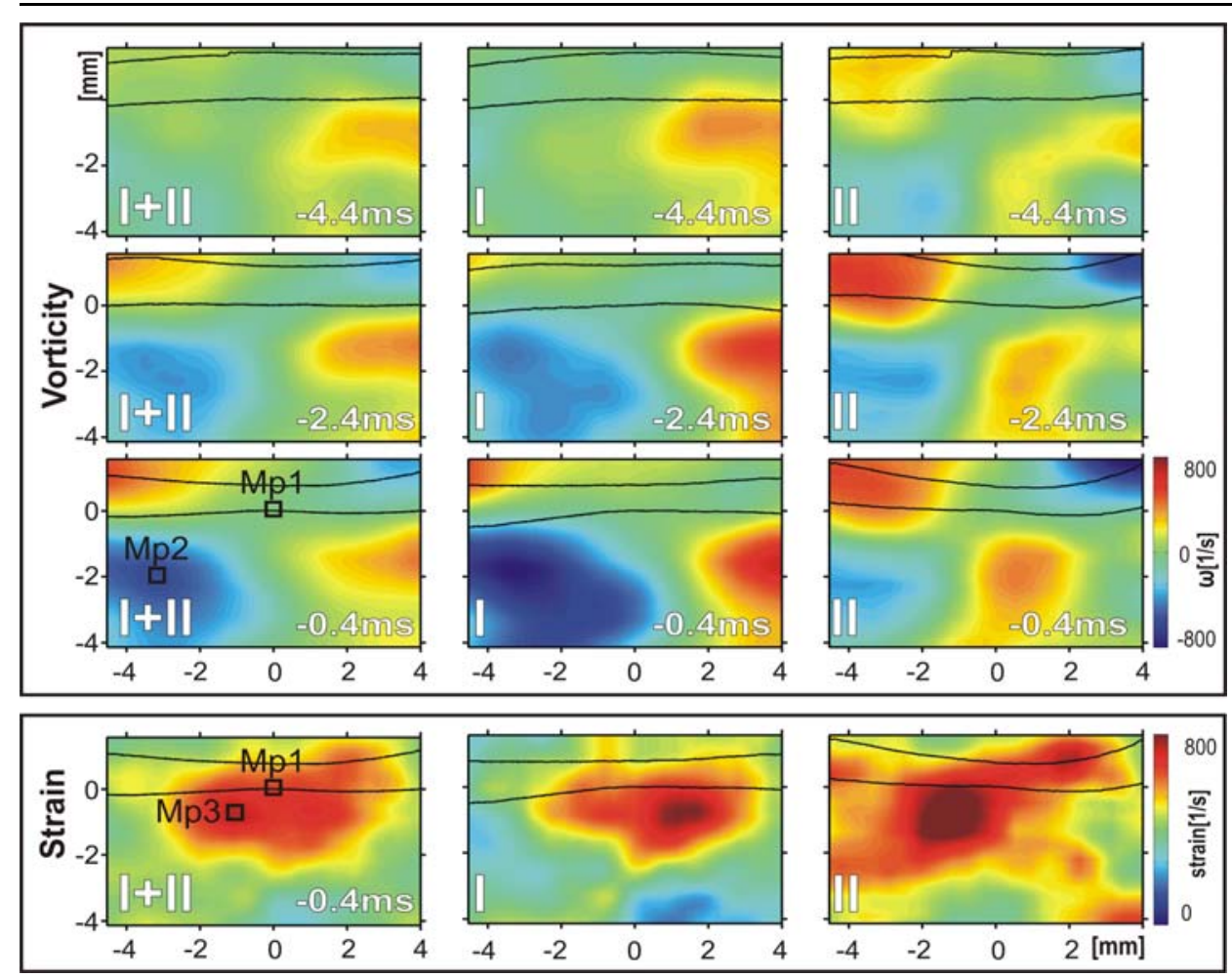

Fig. 4.

Conditional averages of vorticity and strain for all events (I+II), class I and class II events at distinct times prior to extinction.

Double-column width (color print) 


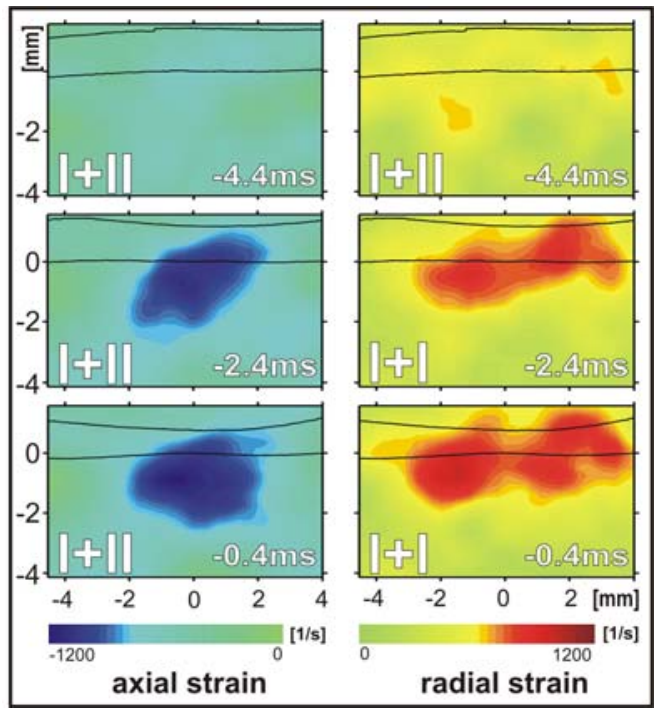

\section{Fig. 5.}

Conditional averages of axial strain (acting compressive) and radial strain (extensive) for all events (I+II) at distinct times prior to extinction.

Single-column width (color print) 


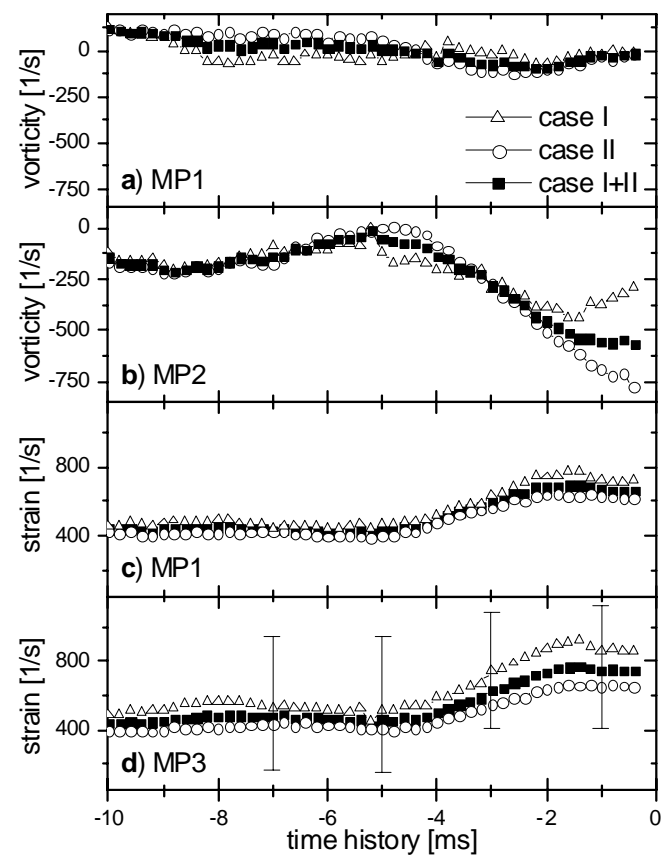

Fig. 6.

Temporal traces of conditional averaged vorticity and strain at monitor points (MP) assigned in Fig. 4.

Single-column width (b/w) 